\title{
ON THE VALUATION OF CITRATE OF IRON AND QUININE.
}

By Frederick W. Fletcher, F.C.S.

THe author said that observations have hitherto been confined, with scarcely an exception, to the determination of the total alkaloid present in the citrate, without regard to its purity as quinine. He had adapted to this salt the process described by Dr. Paul for testing quinine, in the valuable paper which he communicated to the Pharmaceutical Society at an evening meeting in February, 1877. Dr. Paul had stated that a sample of sulphate of quinine, containing an admixture of no less than 30 per cent. of cinchonidine, might, if examined by the Pharmacopoia test, be passed as pure quinine. The author had found 10,15, and even 25 per cent. of sulphate of cinchonidine in foreign quinines, which would, however, pass the Pharmacopœia test. Nor will the Pharmacopœia test detect cinchonidine in citrate of iron and quinine. The alkaloid obtained from the citrate in the manner directed in the Pharmacopœia will dissolve in pure ether, even though 25 per cent. of its weight is cinchonidine. The adaptation of Dr. Paul's plan of fractional crystallisation, which the anthor had devised, is easy of application, and although a considerable quantity of the citrate has to be operated upon, there is little or no loss of quinine, most of the alkaloid being recovered as sulphate. It has, moreover, the advantage of combining three operations in one, as the results indicate :-(1) The exact amount of anhydrous alkaloid; (2) the proportion of the latter which can be converted into crystallisable sulphate of quinine, and (3) the percentage of alkaloids other than quinine.

The operations involved are briefly as follows:-Place 20 grammes of the citrate in 100 c.c. flask, dissolve in 50 c.c. of distilled water, and add gradually an excess of ammonia $(\cdot 960)$, shaking well aftex each addition. This is important, in order that the 
quinine may separate in a state of fine division, as otherwise it is apt to be thrown out in tough lumps, difficult of subsequent solution. Pour in 25 c.c. of washed ether, and agitate with a rotatory motion till the alkaloid has completely dissolved. Transfer the mixture to a small glass separatory funnel, and having run the lower stratum of liquid back into the flask, pour the ethereal solution into 100 c.c. platinum capsule. Treat the liquid in the flask with 20 c.c. more ether, and proceed as before. Repeat this operation a third time. The capsule containing the mixed ethereal solutions is then placed in a saucer of water, and the ether blown off by a current of air from a Fletcher's bellows. This immersion of the capsule in water obviates the tendency of the ether to creep up the sides. The platinum dish, which will now contain a pasty residue, is next placed in the air-bath, previously heated to $120^{\circ}$, and in 15 minutes desiccation is complete. After cooling in an exsiccator, the capsule is covered and removed to the balance. The weight, minus that of the capsule and cover, multiplied by five, is the percentage of total alkaloid. The author had made many hundreds of analyses by this process, and in cases where a determination had been repeated had never found the results vary more than 0.1 per cent. When an estimation of total alkaloid only is required, 2 grammes of citrate is a sufficient quantity to operate upon.

The anlydrous alkaloid is now to be converted into basic sulphate. According to calculation the weight of anhydrous alkaloid in grammes is multiplied by $30 \cdot 86$, and the number of c.c. of decinormal sulphuric acid thus indicated are run into the platinum capsule from a burette, and the former being placed on wire gauze over the flame of a rose burner, the contents are briskly stirred until the alkaloid is all taken up, and a clear solution obtained. This is then transferred to a flask and allowed to cool spontaneously. The crystalline mass which will have formed is thrown on a small calico filter, about 3 inches square, stretched over a beaker, and, when drained, tightly squeezed to remove the last few drops of liquid. The latter is then filtered into a stoppered graduated tube of about 150 c.c. capacity, and its volume noted. Twenty c.c. washed ether and an excess of ammonia are then introduced, and the whole, after being well agitated, set aside for six hours.

In the meantime the squeezed residue is detached from the calico filter, and dried in the air-bath at a temperature of $100^{\circ} \mathrm{C}$. The weight multiplied by 1.18 represents its value as crystallised sulphate of quinine. To this is added the amount of the latter contained in the mother liquor which has been separated (and which may be estimated to contain 1 part in 750), and the total will then represent the proportion of anhydrous alkaloid which can be converted into sulphate.

At the expiration of the time mentioned, the tube which has been set aside is examined, when the cinchonidine and quinidine present will be found to have crystallised out, at the junction of the two liquids. The stratum of ether is removed by a small Nessler pipette; the crystals washed with two successive portions of 10 c.c. of ether, the last few drops of which can be absorbed by a little roll of filter paper. The crystals are then thrown upon a double-tared filter, made, of two papers weighed one against the other, by cutting down the heavier, dried at $120^{\circ}$, and placed on the balance, the outside paper acting as a counterpoise. In practice the author finds that the weight of this first crop of crystals represents on an average two-thirds of the total cinchonidine 
or quinidine present. If, therefore, the amount does not exceed $\cdot 1$ gramme, the percentage of these alkaloids may be estimated to be below 5 per cent. Should the first weighing exceed this limit, the sulphate obtained from the first crystallisation must be dissolved in 100 c.c. boiling water, and treated as before, the weight of allaloid separated by ether being of course added to the amount first obtained.

As thus described, the process may seem tedious, but in reality it is not 80 . Not counting the time which must be allowed for crystallising, the entire estimation may be completed in something under two hours. The method, of course, does not distinguish between quinidine and cinchonidine, the usual tests for which must be applied to the solution of the mixed sulphates. This, however, is a point of minor importance. Two examples will be sufficient to illustrate the working of the process.

Descriptions of experiments were then given, and the author added in conclusion :-

Whether the presence of 5,10 , or 20 per cent. of cinchonidine would bring a sample of citrate of iron and quinine, which nevertheless answcred the B.P. test, within the reach of the Adulteration Act is a point upon which $I$ offer no opinion. An eminent analyst with whom I was lately discussing the point took the negative view. If such is really the case, a revision of the Plarmacopœia will come none too soon. The plausible excuse which is sometimes set up that manufacturers cannot perfectly separate the cinchonidine from the quinine except at a greatly enhanced cost is utterly without foundation. The white sulphate manufactured both by Messrs. Howard and Mr. Whiffen I have never found to give the slightest reaction with Paul's test, and, what is a still more striking fact, the so-called unbleached quinine of the latter maker is frequently quite free from cinchonidine. Certain of the foreign makers are also, as a rule, unimpeachable in this respect, whilst, on the other hand, some others are systematically adulterated.

It may be worth noting, as a matter of practical interest, that the substitution of 1 per cent. of cinchonidine for quinine in the manufacture of citrate of iron and quinine reduces the value $\frac{1}{2} d$. per oz. for each such addition; and when it is remembered that the Pharmacopœia test will easily pass $20 \mathrm{per}$ cent. of cinchonidine, and that the consumption of the citrate is considerably over $100,000 \mathrm{oz}$. per annum, the valuation of this preparation becomes a question of commercial as well as of chemical interest.

\section{ANALYST'S REPORT.}

Tan Adolteration of Mirk.-Dr. C. Meymott Tidy, the medieal ofticer of health for Islington, in his annual report which he has just issued, states that during the year ending Lady-day, 1879, he examined 172 samples of food, and, except in the milk, he found no such evidence of adulteration as warranted him in advising a prosecution. In 12 of the milk cases he gave certificates of adulteration. They were all cases of added water, the adulteration varying on the lowest possible calculation from 10 to 30 per cent. Dr. Tidy adds :- "The milk-sellers have raised the price of their milk, but I can scarcely say that the quality has been improved, the price being, in fact, no guarantee of genuineness. The extent to which milk is adulterated is serious. For we must remember that to many (and those for the most part the very old and the very young) milk constitutes the sole article of diet. The doctor can easily be thwarted in his treatment of a patient by the dairyman. The public are in this way cheated of their money; and if that were all it would be bad enough, but they are cheated of their health too. And yet during this year only a single sample of food was submitted to me by the public for examination, and that one article was not milk, but whisky." In concluding his report Dr. Tidy says :-Would it not 
be worth while, remembering that there are yet so many things to be attended to, and so many reforms needed, in respect chiefly to the drinking habits of the people and their method of life, for sanitarians to turn their attention for a few years to these sadly-neglected topics, instead of confining their thoughts almost exclusively, as they have been doing of late years, to the water as the source of all ills and miseries? It is not simply the time wasted on the subject that is to be deplored, but it is the unnecessary alarm the discussion of such subjects excites in the public mind." 